\title{
On some developments of the Erdős-Ginzburg-Ziv Theorem II
}

\author{
by \\ Arie Bialostocki (Moscow, ID), Paul Dierker (Moscow, ID), \\ DAVID GRYNKIEWICZ (Pasadena, CA) and \\ MARK Lotspeich (Caldwell, ID)
}

1. Introduction. Let $S$ be a sequence of elements from the cyclic group $\mathbb{Z}_{m}$. We say $S$ is zsf (zero-sum free) if there does not exist an $m$-term subsequence of $S$ whose sum is zero. Let $g(m, k)$ (resp. $g^{*}(m, k)$ ) denote the least integer such that every sequence $S$ with at least (resp. with exactly) $k$ distinct elements and length $g(m, k)$ (resp. $g^{*}(m, k)$ ) must contain an $m$-term subsequence whose sum is zero. By an affine transformation in $\mathbb{Z}_{m}$ we mean a map of the form $x \mapsto a x+b$, with $a, b \in \mathbb{Z}_{m}$ and $\operatorname{gcd}(a, m)=1$. Furthermore, let $E(m, s)$ denote the set of all equivalence classes of zsf sequences $S$ of length $s$, up to order and affine transformation, that are not a proper subsequence of another zsf sequence. Using the above notation, the renowned Erdös-Ginzburg-Ziv Theorem ([1], [11]) states that $g(m, 1)=g(m, 2)=2 m-1$ for $m \geq 2$.

The function $g(m, k)$ was introduced in [4], where it was shown that $g(m, 4)=2 m-3$ for $m \geq 4$. Furthermore, based on a lower bound construction the authors conjectured the value of $g(m, k)$ for fixed $k$ and sufficiently large $m$. Concerning the upper bound, they established an upper bound for $m$ prime modulo the affirmation of the Erdös-Heilbronn conjecture (EHC). Since then, the EHC has been affirmed [9], [2], moreover, the bound given in [4] was extended for nonprimes in [19]. As will later be seen, it is worthwhile to mention that the affirmation of the EHC has resulted in several attempted generalizations and related results [6], [15], [22],[25]. Other relevant developments concerning $g(m, k)$ appear in [3], [5], [7], [13], [14], [16], [23]. For example, the value $g(m, 3)=2 m-2$ was determined in [3], and the closely related function $g^{*}(m, k)$, introduced in [5] and further investigated in [4], [14], [16], was determined for all $m$ and $k$ satisfying $k>m / 2+1$.

2000 Mathematics Subject Classification: Primary 11B75; Secondary 11B50, 05D10. 
This paper started under the authorship of the first, second and fourth authors, and was cited in [4] as in preparation. Actually, a rough draft was ready determining $g(m, 5)$ using the methods of [4]. The motivation for the current paper is twofold. First, the third author was able to determine exactly $g(m, k)$ for fixed $k$ and large $m$ by improving the known lower bound construction and adapting the proof used by Gao and Hamidoune [19] to obtain a better upper bound than the one conjectured in [4]. Consequently, the following conjecture of [4] has been affirmed.

Conjecture 1.1. For every $k \geq 2$, there exists an integer $m_{0}=m_{0}(k)$ such that if $m>m_{0}$, then

(a) $g(m, k)=2 m-c$, where $c=c(k)$ is independent of $m$,

(b) $g^{*}(m, k)=g(m, k)$.

Thus, the $g(m, k)$ problem for fixed $k$ and large $m$ has been put to rest. Second, as several recent works, e.g. [21], [27], use the known values of $g(m, k)$ and $E(m, s)$ for $k \leq 4$ and $s \geq 2 m-3$, and as it is likely that $g(m, 5)$ will be needed for further zero-sum applications, we determine $g(m, 5)$ for every $m \geq 5$.

The paper is organized as follows. In Section 2, definitions and notation are introduced and known results needed later in the paper are listed. In Section 3, first the upper bound proof of [19] is adapted to find, for a sequence $S$ with $|S| \geq 2 m-\lfloor m / 4\rfloor-2$, necessary and sufficient conditions in terms of a system of inequalities over the integers for $S$ to be zsf. This result and a lower bound construction imply the value of $g(m, k)$ for fixed $k$ and large $m$. Following is the affirmation of Conjecture 1.1. Section 4 contains the evaluation of $g(m, 5)$ for every $m \geq 5$. The paper concludes with an appendix listing the elements of $E(m, s)$ for every $m$ and $s$ satisfying $2 m-2 \geq s \geq \max \{2 m-8,2 m-\lfloor m / 4\rfloor-2\}$.

2. Preliminaries. Let $G$ denote an abelian group of order $m$. As we work simultaneously with the cyclic group $\mathbb{Z}_{m}$ of residue classes modulo $m$ and the additive group of the integers $\mathbb{Z}$, for $\alpha \in \mathbb{Z}_{m}$ we denote by $\bar{\alpha}$ the least positive integer that is congruent to $\alpha$ modulo $m$. A sequence $S$ of elements from $G$ is abbreviated as a string using exponential notation (e.g. the sequence $1,1,1,2,2,2,2$ is abbreviated by $1^{3} 2^{4}$ ). Furthermore, the length of $S$ is denoted by $|S|$. Let $t$ be a nonnegative integer. Denote by $\sum S$ the set of all sums over nonempty subsequences of $S$, and by $\sum_{t} S$ (resp. $\sum_{\geq t} S$ ) the set of all sums over subsequences of $S$ of length exactly (resp. at least) $t$. If $A, B \subseteq G$, then their sumset, $A+B$, is the set of all possible pairwise sums, i.e. $\{a+b \mid a \in A, b \in B\}$.

Following Kemperman [24], a set $A \subseteq G$ is $H$-periodic if it is the union of $H$-cosets for some nontrivial subgroup $H \leq G$. Furthermore, an n-set 
partition of $S$ is a sequence of $n$ nonempty subsequences of $S$, pairwise disjoint as sequences, such that every term of $S$ belongs to exactly one subsequence, and the terms in each subsequence are distinct. Thus such subsequences can be considered sets. Finally let $\varphi$ be the map that takes a sequence to its underlying set (e.g. $\varphi(1,1,1,2,2,4)=\{1,2,4\})$.

First, we state three well known theorems: the first one is a generalized version of what is known as the Caveman Theorem [12], followed by a generalized form of the Erdös-Ginzburg-Ziv (EGZ) Theorem [1], [11], and the affirmed EHC [2], [9]. The original EGZ Theorem is Theorem 2.2 with $r=1$; and Theorem 2.2 is obtained by $r$ applications of the EGZ Theorem. Theorem 2.1 is similarly obtained, where the original Caveman Theorem is the case $|S|=m$.

THEOREM 2.1. Let $S$ be a sequence of elements from an abelian group $G$ of order $m$. If $|S| \geq m$, then there exists a subsequence of $S$ of length $r$ whose terms sum to zero, where $r$ satisfies $|S|-(m-1) \leq r \leq|S|$.

THEOREM 2.2. Let $r$ be a positive integer. If $S$ is a sequence of $(r+1) m-1$ elements from an abelian group $G$ of order $m$, then $S$ contains an rm-term subsequence which sums to zero.

THEOREM 2.3. Let $S$ be a sequence of $k$ distinct elements from $\mathbb{Z}_{m}$. If $m$ is prime, then $\left|\sum_{h} S\right| \geq \min \left\{m, h k-h^{2}+1\right\}$.

The following two theorems of Gao [17], [18], respectively, are central to the proof of Theorem 3.1.

THEOREM 2.4. Let $l$ and $m$ be positive integers satisfying $2 \leq l \leq$ $\lfloor m / 4\rfloor+2$, and let $S$ be a sequence of elements from $\mathbb{Z}_{m}$ satisfying $|S|=$ $2 m-l$. If $0 \notin \sum_{m} S$, then up to order and affine transformation, $S=$ $0^{u} 1^{v} c_{1} \ldots c_{w}$, where $m-2 l+3 \leq v \leq u \leq m-1$ and $w \leq l-2$.

ThEOREM 2.5. Let $m, n$ and $h$ be positive integers. Suppose $G$ is a finite abelian group of order $m$, and $g \in G$. Furthermore, let $S=0^{h} a_{1} \ldots a_{n}$ be $a$ sequence of elements from $G$ such that the multiplicity of every element in the subsequence $T=a_{1} \ldots a_{n}$ is at most $h$. If $g \in \sum_{\geq m-h} T$, then $g \in \sum_{m} S$.

Next, we state the Cauchy-Davenport Theorem [8], [26], and a recently proved composite analog of it [20].

THEOREM 2.6. For positive integers $n$ and $m$, let $A_{1}, \ldots, A_{n} \subseteq \mathbb{Z}_{p}$. If $m$ is prime, then

$$
\left|\sum_{i=1}^{n} A_{i}\right| \geq \min \left\{m, \sum_{i=1}^{n}\left|A_{i}\right|-n+1\right\} .
$$

THEOREM 2.7. Let $n$ be an integer and let $S$ be a sequence of elements from an abelian group $G$ of order $m$ such that $|S| \geq n$ and every element of 
$S$ appears at most $n$ times in $S$. Furthermore, let $p$ be the smallest prime divisor of $m$. Then either

(i) there exists an $n$-set partition, $A_{1}, \ldots, A_{n}$, of $S$ such that

$$
\left|\sum_{i=1}^{n} A_{i}\right| \geq \min \left\{m,(n+1) p, \sum_{i=1}^{n}\left|A_{i}\right|-n+1\right\},
$$

or

(ii) there exists $\alpha \in G$ and a nontrivial proper subgroup $H$ of index $a$, such that all but at most $a-2$ terms of $S$ are from the coset $\alpha+H$, and there exists an $n$-set partition, $A_{1}, \ldots, A_{n}$, of the subsequence of $S$ consisting of terms of $S$ from $\alpha+H$ such that $\sum_{i=1}^{n} A_{i}=n \alpha+H$.

When applying the above theorem, the following two basic propositions about $n$-set partitions and sumsets are often used, and for the sake of clarity, we provide their proofs.

Proposition 2.1. A sequence $S$ has an $n$-set partition $A$ if and only if the multiplicity of each element in $S$ is at most $n$ and $|S| \geq n$. Furthermore, a sequence $S$ with an $n$-set partition has an $n$-set partition $A^{\prime}=A_{1} \ldots A_{n}$ such that ||$A_{i}|-| A_{j}|| \leq 1$ for all $i$ and $j$ satisfying $1 \leq i \leq j \leq n$.

Proof. Suppose $S$ has an $n$-set partition. Then from the definition the multiplicity of each element in $S$ is at most $n$, and since empty sets are not allowed in the $n$-set partition, it follows that $|S| \geq n$. Next suppose that the multiplicity of each element in $S$ is at most $n$ and $|S| \geq n$. Let $\varphi(S)=\left\{s_{1}, \ldots, s_{u}\right\}$, and rearrange the terms of $S$ so that all the terms that are equal to $s_{1}$ come first, followed by all the terms that are equal to $s_{2}$, and so forth, terminating with the terms equal to $s_{u}$. Let us denote this new sequence by $S^{\prime}=x_{1} x_{2} \ldots x_{k n+r}$, where $|S|=\left|S^{\prime}\right|=k n+r$ and $0 \leq r<n$. Consider the following sequence $A$ of $n$ subsequences of $S^{\prime}$ written vertically:

$$
A=\left(\begin{array}{c}
x_{1} \\
x_{n+1} \\
\vdots \\
x_{(k-1) n+1} \\
x_{k n+1}
\end{array}\right) \cdots\left(\begin{array}{c}
x_{r} \\
x_{n+r} \\
\vdots \\
x_{(k-1) n+r} \\
x_{k n+r}
\end{array}\right)\left(\begin{array}{c}
x_{r+1} \\
x_{n+r+1} \\
\vdots \\
x_{(k-1) n+r+1} \\
\cdot
\end{array}\right) \cdots\left(\begin{array}{c}
x_{n} \\
x_{2 n} \\
\vdots \\
x_{k n} \\
\cdot
\end{array}\right) .
$$

We will show that $A$ is an $n$-set partition of $S^{\prime}$ and hence of $S$. Indeed, since $|S| \geq n$, it follows that none of the sets in $A$ are empty. Furthermore, in view of the definition of $S^{\prime}$ and the fact that the maximum multiplicity of a term in $S^{\prime}$ does not exceed $n$, it follows that $x_{j_{1} n+i} \neq x_{j_{2} n+i}$, for every $i$ and every $j_{1} \neq j_{2}$. Thus $A$ is an $n$-set partition of $S$. The furthermore part is clear from the definition of $A$. 
Proposition 2.2. Let $S$ be a sequence of elements from a finite abelian group $G$, and let $A=A_{1} \ldots A_{n}$ be an $n$-set partition of $S$, where $\left|\sum_{i=1}^{n} A_{i}\right|=r$, and $s$ is the cardinality of the largest set in $A$. Furthermore, let $a_{1} \ldots a_{n}$ be a subsequence of $S$ such that $a_{i} \in A_{i}$ for $i=1, \ldots, n$.

(i) There exists a subsequence $S^{\prime}$ of $S$ and an $n^{\prime}$-set partition $A^{\prime}=$ $A_{1}^{\prime} \ldots A_{n^{\prime}}^{\prime}$ of $S^{\prime}$, which is a subsequence of the $n$-set partition $A=A_{1} \ldots A_{n}$, such that $n^{\prime} \leq r-s+1$ and $\left|\sum_{i=1}^{n^{\prime}} A_{i}^{\prime}\right|=r$.

(ii) There exists a subsequence $S^{\prime}$ of $S$ of length at most $n+r-1$, and an $n$-set partition $A^{\prime}=A_{1}^{\prime} \ldots A_{n^{\prime}}^{\prime}$ of $S^{\prime}$, where $A_{i}^{\prime} \subseteq A_{i}$ for $i=1, \ldots, n$, such that $\sum_{i=1}^{n} A_{i}^{\prime}=\sum_{i=1}^{n} A_{i}$. Furthermore, $a_{i} \in A_{i}^{\prime}$ for $i=1, \ldots, n$.

Proof. We first prove (i). Assume without loss of generality that $\left|A_{1}\right|=s$. We will construct the $n^{\prime}$-set partition $A^{\prime}$ in $n$ steps as follows; and $S^{\prime}$ will be implied implicitly. Denote by $A^{(k)}=A_{1}^{\prime} \ldots A_{a_{k}}^{\prime}$ the sequence constructed after $k$ steps, and hence $A^{\prime}=A^{(n)}$ and $n^{\prime}=a_{n}$. Let $A^{(1)}=A_{1}$, and for $k=1, \ldots, n-1$, let

$$
A^{(k+1)}= \begin{cases}A^{(k)} & \text { if }\left|\sum_{i=1}^{a_{k}} A_{i}^{\prime}+A_{k+1}\right|=\left|\sum_{i=1}^{a_{k}} A_{i}^{\prime}\right|, \\ A^{(k)} A_{k+1} & \text { if }\left|\sum_{i=1}^{a_{k}} A_{i}^{\prime}+A_{k+1}\right|>\left|\sum_{i=1}^{a_{k}} A_{i}^{\prime}\right| .\end{cases}
$$

It is easily seen by the above algorithm that $\left|\sum_{i=1}^{a_{n}} A_{i}^{\prime}\right|=\left|\sum_{i=1}^{n} A_{i}\right|=r$. Furthermore, since each kept term increases the cardinality of the sumset of the previous terms of $A^{\prime}$ by at least one, and since $\left|A_{1}\right|=s$, it follows that at most $r-s$ terms, excluding $A_{1}$, were kept, and thus $a_{n}=n^{\prime} \leq 1+r-s$.

The proof of (ii) is similar to that of (i). First, for $i=1, \ldots, n$, let the elements of $A_{i}$ be $\left\{a_{1}^{(i)}, \ldots, a_{\left|A_{i}\right|}^{(i)}\right\}$, where $a_{1}^{(i)}=a_{i}$. We will construct the $n$ set partition $A^{\prime}$ in a two loop algorithm. The outer loop has $n$ steps, where at the $i$ th step the set $A_{i}^{\prime}$ is constructed using the inner loop. In turn, the inner loop, at the $i$ th step of the outer loop, constructs $A_{i}^{\prime}$ in $\left|A_{i}\right|$ steps. For a given $i$, where $1 \leq i \leq n$, let $A_{i}^{(k)}$ denote the set constructed after $k$ steps of the inner loop at the $i$ th step of the outer loop, and hence $A^{\prime}=A_{1}^{\left(\left|A_{1}\right|\right)} \ldots A_{n}^{\left(\left|A_{n}\right|\right)}$ with $S^{\prime}$ implied implicitly. For a given $j$, where $1 \leq j \leq n$, let $A_{j}^{(1)}=\left\{a_{j}\right\}$, and for $k=1, \ldots,\left|A_{j}\right|-1$, let

$$
A_{j}^{(k+1)}=\left\{\begin{array}{c}
A_{j}^{(k)} \quad \text { if }\left|\sum_{i=1}^{j-1} A_{i}^{\left(\left|A_{i}\right|\right)}+A_{j}^{(k)}\right|=\left|\sum_{i=1}^{j-1} A_{i}^{\left(\left|A_{i}\right|\right)}+\left(A_{j}^{(k)} \cup\left\{a_{k+1}^{(j)}\right\}\right)\right|, \\
A_{j}^{(k)} \cup\left\{a_{k+1}^{(j)}\right\} \\
\quad \text { if }\left|\sum_{i=1}^{j-1} A_{i}^{\left(\left|A_{i}\right|\right)}+A_{j}^{(k)}\right|<\left|\sum_{i=1}^{j-1} A_{i}^{\left(\left|A_{i}\right|\right)}+\left(A_{j}^{(k)} \cup\left\{a_{k+1}^{(j)}\right\}\right)\right| .
\end{array}\right.
$$

It is easily seen by the above algorithm that $A_{i}^{\left(\left|A_{i}\right|\right)} \subseteq A_{i}$ and $a_{i} \in$ $A_{i}^{\left(\left|A_{i}\right|\right)}$ for $i=1, \ldots, n$, and that $\left|\sum_{i=1}^{n} A_{i}^{\left(\left|A_{i}\right|\right)}\right|=\left|\sum_{i=1}^{n} A_{i}\right|=r$; and since $\sum_{i=1}^{n} A_{i}^{\left(\left|A_{i}\right|\right)} \subseteq \sum_{i=1}^{n} A_{i}$, it follows that $\sum_{i=1}^{n} A_{i}^{\left(\left|A_{i}\right|\right)}=\sum_{i=1}^{n} A_{i}$. Further- 
more, since each kept element $a_{k}^{(j)}$, where $k \neq 1$ if $j \neq 1$, increases the cardinality of the sumset by at least one, it follows that at most $r-1$ terms, excluding the $a_{i}$ 's, were kept, and hence $\left|S^{\prime}\right| \leq n+r-1$.

We will also need the following theorem of [16].

Theorem 2.8. Let $m$ and $k$ be integers with $m \geq k \geq 2$ and $m \geq 5$.

(a) If $m / 2+1<k \leq m-1$, then $g(m, k)=m+2$.

(b) If $k=m$, then $g(m, k)= \begin{cases}m, & m \text { odd, } \\ m+1, & m \text { even. }\end{cases}$

We conclude the preliminaries with a theorem of Eggleton and Erdős [10].

TheOREM 2.9. Let $S$ be a sequence of $k$ distinct elements from a finite abelian group. If $0 \notin \sum S$ and $k \geq 4$, then $\left|\sum S\right| \geq 2 k$.

3. A theorem of Gao and Hamidoune revisited. Theorem 3.1 gives necessary and sufficient conditions for a sequence $S$ of sufficient length to be zsf. More precisely, it reduces the problem of determining extremal zsf sequences of sufficient length to the problem of finding integer partitions with a fixed number of parts and all parts greater than 1. Its proof is an adaption of a proof of Gao and Hamidoune [19].

TheOREm 3.1. For integers $m$ and $l$, let $S$ be a sequence of elements from $\mathbb{Z}_{m}$, satisfying $|S|=2 m-l \geq 2 m-\lfloor m / 4\rfloor-2$. The sequence $S$ does not contain an m-term zero-sum subsequence if and only if there exists a sequence $S^{\prime}=0^{u} 1^{v} a_{1} \ldots a_{w_{1}} b_{1} \ldots b_{w_{2}}$, where $1<\bar{a}_{i} \leq m / 2$ and $1 \leq \overline{-b_{i}}<$ $m / 2$, that is equivalent to $S$ up to order and affine transformation, and for which the following four inequalities are satisfied:

$$
\begin{aligned}
& \sum_{i=1}^{w_{1}} \bar{a}_{i} \leq m-v-1 \quad \text { and } \sum_{i=1}^{w_{2}} \overline{-b_{i}} \leq m-u-1-w_{2}, \\
& m-2 l+3 \leq v \leq u \leq m-1 \text { and } w_{1}+w_{2} \leq l-2 .
\end{aligned}
$$

Moreover, equality holds in both inequalities of (1) if and only if $S$ belongs to an equivalence class of $E(m, 2 m-l)$.

Proof. First, suppose $S$ is a sequence of elements from $\mathbb{Z}_{m}$, satisfying $|S|=2 m-l \geq 2 m-\lfloor m / 4\rfloor-2$, and $0 \notin \sum_{m} S$. Hence from Theorem 2.4 it follows that $S$ is equivalent, up to order and affine transformation, to a sequence $S^{\prime}=0^{u} 1^{v} a_{1} \ldots a_{w_{1}} b_{1} \ldots b_{w_{2}}$ satisfying the inequalities in (2), where $1<\bar{a}_{i} \leq m / 2$ and $1 \leq \overline{-b_{i}}<m / 2$. Since the fact that $S$ is zsf implies that $S^{\prime}$ is zsf, it follows from Theorem 2.5 that for any given subsequence $T$ of $a_{1} \ldots a_{w_{1}} b_{1} \ldots b_{w_{2}}$,

$$
\text { either } \overline{\sum_{t_{i} \in T} t_{i}} \leq m-v-1 \quad \text { or } \quad \overline{\sum_{t_{i} \in T} t_{i}} \geq u+1+|T|
$$


and

$$
\text { either } \overline{-\sum_{t_{i} \in T} t_{i}} \leq m-u-1-|T| \quad \text { or } \quad \overline{-\sum_{t_{i} \in T} t_{i}} \geq v+1 .
$$

Induction on $r$, in view of (3) and the following three inequalities (i) $l \leq$ $\lfloor m / 4\rfloor+2$, (ii) $m-v-1 \leq\lfloor m / 2\rfloor$ (follows from (2) and $l \leq\lfloor m / 4\rfloor+2$ ), (iii) $3 m-4 l+5 \leq u+2 v$ (follows from (2)), implies

$$
\sum_{i=1}^{r} \bar{a}_{i}=\overline{\sum_{i=1}^{r} a_{i}} \leq m-v-1
$$

for every $r$ satisfying $1 \leq r \leq w_{1}$.

Similarly, induction on $r$, in view of (4) and the inequalities (i)-(iii), and the fact that $u \geq v$, implies

$$
\sum_{i=1}^{r} \overline{-b_{i}}=\overline{-\sum_{i=1}^{r} b_{i}} \leq m-u-1-r
$$

for every $r$ satisfying $1 \leq r \leq w_{2}$. Hence (5) and (6) imply (1).

Next suppose $S$ is an arbitrary sequence of residues from $\mathbb{Z}_{m}$ that satisfies (1) and (2). Actually, we will use only the fact that (1) is satisfied and $v \leq u \leq m-1$. It follows from (1) that any $m$-term zero-sum modulo $m$ subsequence of $S$ must be zero-sum in $\mathbb{Z}$ as well. In addition, it follows from (1) that the longest zero-sum in $\mathbb{Z}$ subsequence of $S$ that does not contain a zero is of length $w_{2}+\sum_{i=1}^{w_{2}} \overline{-b_{i}} \leq m-u-1$. Hence any $m$-term zero-sum subsequence must use at least $u+1$ zeros, which exceeds the multiplicity of zero in $S$. Thus $S$ is zsf, and as affine transformations and reordering preserve $m$-term zero-sum subsequences, the proof of the main part of the theorem is complete. Notice that the two inequalities in (1) are interchanged by the affine transformation which interchanges 0 and 1 . Hence, the moreover part of the theorem is easily deduced from the main part of the theorem.

THEOREM 3.2. Let $m \geq k \geq 2$ be positive integers. If $k$ is odd and $m \geq$ $\left(k^{2}+4 k+3\right) / 8+1$ or $k$ is even and $m \geq\left(k^{2}+2 k\right) / 8+1$, then $g^{*}(m, k) \geq$ $2 m-\left\lfloor\left(k^{2}-2 k+5\right) / 4\right\rfloor$.

Proof. If $k$ is even, consider the sequence

$$
S_{0}=\left(-\frac{k-2}{2}\right) \ldots(-1)(0)^{m-\left(k^{2}+2 k\right) / 8}(1)^{m-\left(k^{2}+2 k\right) / 8}(2) \ldots\left(\frac{k}{2}\right),
$$

and if $k$ is odd, consider the sequence

$$
S_{1}=\left(-\frac{k-3}{2}\right) \ldots(-1)(0)^{m-\left(k^{2}-1\right) / 8}(1)^{m-\left(k^{2}+4 k+3\right) / 8}(2) \ldots\left(\frac{k+1}{2}\right) .
$$

It follows from the hypotheses that both strings are well defined. Since both $S_{1}$ and $S_{2}$ satisfy (1), and since $v \leq u \leq m-1$, where $u$ and $v$ are the 
multiplicities of 0 and 1 respectively, it follows from the proof of the second direction of Theorem 3.1 that $S_{1}$ and $S_{2}$ are zsf.

We conclude the section with Theorem 3.3, which determines the value of $g(m, k)$ for fixed $k$ and sufficiently large $m$, disproving Conjecture 5.1 of [4], and proving Conjectures 1.1(a) and 1.1(b) in parts (a) and (b) respectively. Again, its proof is an adaptation of the proof in [19].

TheOREM 3.3. Let $m \geq k \geq 2$ be positive integers. If $k$ is even and $m \geq k^{2}-2 k-4$ or $k$ is odd and $m \geq k^{2}-2 k-3$, then

(a) $g(m, k)=2 m-\left\lfloor\left(k^{2}-2 k+5\right) / 4\right\rfloor$,

(b) $g^{*}(m, k)=g(m, k)$.

Proof. From Theorem 3.2, and from the trivial fact that $g^{*}(m, k) \leq$ $g(m, k)$, it suffices for both parts (a) and (b) to show $g(m, k) \leq 2 m-$ $\left\lfloor\left(k^{2}-2 k+5\right) / 4\right\rfloor$. Assume to the contrary that there is a sequence $S$ of elements from $\mathbb{Z}_{m}$ with $|S|=2 m-\left\lfloor\left(k^{2}-2 k+5\right) / 4\right\rfloor$ and $0 \notin \sum_{m} S$. From the hypotheses and the fact that $k^{2} \equiv 0$ or $1 \bmod 4$, it follows that $\left\lfloor\left(k^{2}-2 k+5\right) / 4\right\rfloor \leq\lfloor m / 4\rfloor+2$. Hence from Theorem 3.1 it follows that without loss of generality $S$ satisfies (1) and (2). Let $c_{1}=\left|\left\{a_{1}, \ldots, a_{w_{1}}\right\}\right|$ and $c_{2}=\left|\left\{b_{1}, \ldots, b_{w_{2}}\right\}\right|$. It follows from the first inequality in (1) that $2+3+\ldots+\left(c_{1}+1\right)+2\left(w_{1}-c_{1}\right) \leq m-v-1$, implying that

$$
\frac{c_{1}^{2}-c_{1}}{2}+2 w_{1} \leq m-v-1 \text {. }
$$

Likewise from the second inequality in (1), it follows that

$$
\frac{c_{2}^{2}-c_{2}}{2}+2 w_{2} \leq m-u-1-w_{2}
$$

Inequalities (7) and (8) imply

$$
\frac{c_{1}^{2}-c_{1}}{2}+\frac{c_{2}^{2}-c_{2}}{2} \leq m-v-1-w_{1}+m-u-1-w_{2}=l-2,
$$

which, in turn, yields

$l \geq \frac{\left(c_{1}+c_{2}\right)^{2}}{4}+\frac{c_{1}+c_{2}}{2}+2 \geq \frac{(k-2)^{2}}{4}+\frac{k-2}{2}+2=\frac{k^{2}-2 k+4}{4}+1>l$,

which is a contradiction; and the proof is complete.

4. The Erdös-Heilbronn conjecture and $g(m, 5)$. In view of Theorem $3.3, g(m, 5)$ has been determined for $m \geq 12$. In this section, we present an abbreviated proof determining $g(m, 5)$ for all $m \geq 5$. We will make use of the following conjecture, which can be verified for $k \leq 5$ with some effort by considering the equations generated by the 2 -sums of a 5 -set $S$ with $\left|\sum_{2} S\right|<7$. 
CONJECTURE 4.1. Let $S$ be a sequence of $k \geq 2$ distinct elements from $\mathbb{Z}_{m}$. If $\left|\sum_{2} S\right|<2 k-3$, then either $\sum_{h} S$ is $H$-periodic, where $|H|>2$, or $S$ is $K$-periodic, where $|K|=2$.

TheOREM 4.1. Let $m \geq 5$. Then $g(6,5)=8$, and if $m \neq 6$, then $g(m, 5)=2 m-5$.

Proof. For $m \leq 6$ the result follows from Theorem 2.8. Suppose $S$ is zsf and $|S|=2 m-5$. We may assume that 0 has the greatest multiplicity in $S$.

CAse 1: The multiplicity of 0 in $S$ is at most $m-2$. Applying Conjecture 4.1 with $k=5$ to all possible 5 -sets of $\varphi(S)$ that include 0 , we can either find a 5-set $A \subseteq \varphi(S)$ such that $\left|\sum_{2} A\right|=\left|\sum_{3} A\right| \geq 7$ and $0 \in A$, or else there exists a subgroup $H$ of cardinality $h=5$ or $h=6$ such that $\varphi(S) \subseteq H$. In the latter case, $m \geq 10$, and so from Theorem 2.2 it follows that any subsequence with length $m+h-1 \leq 2 m-5$ must contain an $m$-term zero-sum subsequence, a contradiction. So $\sum_{3} A \geq 7$. In view of the assumption of the case and Proposition 2.1, there exists an $(m-3)$-set partition $P$ of $S \backslash A$ with $m-7$ sets of cardinality two. Applying Theorem 2.7 to $S \backslash A$, and using $\sum_{3} A \geq 7$, we obtain an $m$-term zero-sum subsequence of $S$, provided conclusion (i) of Theorem 2.7 holds. Hence we are done for $m \leq 8$.

So assume that conclusion (ii) of Theorem 2.7 holds with coset $\alpha+H$ of index $a$, and without loss of generality assume $\alpha=0$. Let $P$ be the $(m-3)$-set partition implied by conclusion (ii) of Theorem 2.7. Applying Proposition 2.2(i) followed by Proposition 2.2(ii) to $P$ we obtain an $(m / a-1)$-set partition $P^{\prime}$ of a subsequence $Q$ of $S \backslash A$ of length at most $2 m / a-2$, whose sumset is also $H$. Then there exists a subsequence $R$ of $S \backslash A$ of length $a-1$ whose terms are from $H$ and are not used in $P^{\prime}$. We can apply Theorem 2.1 to a subsequence of $S \backslash\{Q \cup R\}$ of length $m-m / a+1$ with its terms considered as elements from $\mathbb{Z}_{m} / H$ to obtain a subsequence $T$ of $S \backslash\{Q \cup R\}$ whose sum is an element of $H$ and of length $r$, where $r$ satisfies $m-m / a-a+2 \leq r \leq m-m / a+1$. Since the sumset of $P^{\prime}$ is $H$, we can find $m / a-1$ terms from $P^{\prime}$ which along with $T$ and an appropriate number of terms from $R$ give an $m$-term subsequence with sum zero.

CAsE 2: The multiplicity of 0 in $S$ is $m-1$. Let $T^{\prime}$ be a subsequence of $S$ that consists of 4 distinct nonzero residue classes and 3 zeros. In view of Proposition 2.1, it follows that there exists an $(m-4)$-set partition $P^{\prime}$ of $S \backslash T^{\prime}$ with $m-8$ cardinality two sets. Applying Theorem 2.7 to $P$ and Theorem 2.9 to $\varphi\left(T^{\prime}\right) \backslash\{0\}$, we find an $m$-term zero-sum subsequence provided conclusion (i) of Theorem 2.7 holds. If conclusion (ii) of Theorem 2.7 holds instead, then since $m-4>a-2$ implies $0 \in \alpha+H$, the arguments from the end of Case 1 complete the proof. 
Appendix. In the following table, Theorem 3.1 is used to list the values of $E(m, s)$ for all $m$ and $s$ satisfying $2 m-2 \geq s \geq \max \{2 m-8$, $2 m-\lfloor m / 4\rfloor-2\}$.

\begin{tabular}{|c|c|c|c|c|}
\hline$m$ & $s$ & \multicolumn{3}{|c|}{$E(m, s)$} \\
\hline$m \geq 2$ & $2 m-2$ & $0^{m-1} 1^{m-1}$ & & \\
\hline$m \geq 4$ & $2 m-3$ & $0^{m-1} 1^{m-3} 2$ & & \\
\hline$m \geq 8$ & $2 m-4$ & $0^{m-1} 1^{m-5} 2^{2}$ & $(-1) 0^{m-3} 1^{m-3} 2$ & $0^{m-1} 1^{m-4} 3$ \\
\hline$m \geq 12$ & $2 m-5$ & $\begin{array}{l}0^{m-1} 1^{m-7} 2^{3} \\
(-1) 0^{m-3} 1^{m-4} 3\end{array}$ & $\begin{array}{l}(-1) 0^{m-3} 1^{m-5} 2^{2} \\
0^{m-1} 1^{m-5} 4\end{array}$ & $0^{m-1} 1^{m-6} 23$ \\
\hline$m \geq 16$ & $2 m-6$ & $\begin{array}{l}0^{m-1} 1^{m-9} 2^{4} \\
0^{m-1} 1^{m-8} 2^{2} 3 \\
0^{m-1} 1^{m-7} 24 \\
(-2) 0^{m-4} 1^{m-4} 3\end{array}$ & $\begin{array}{l}(-1) 0^{m-3} 1^{m-7} 2^{3} \\
(-1) 0^{m-3} 1^{m-6} 23 \\
0^{m-1} 1^{m-7} 3^{2} \\
0^{m-1} 1^{m-6} 5\end{array}$ & $\begin{array}{l}(-1)^{2} 0^{m-5} 1^{m-5} 2^{2} \\
(-2) 0^{m-4} 1^{m-5} 2^{2} \\
(-1) 0^{m-3} 1^{m-5} 4\end{array}$ \\
\hline$m \geq 20$ & $2 m-7$ & $\begin{array}{l}0^{m-1} 1^{m-11} 2^{5} \\
0^{m-1} 1^{m-10} 2^{3} 3 \\
(-1)^{2} 0^{m-5} 1^{m-6} 23 \\
(-1) 0^{m-3} 1^{m-7} 24 \\
(-1)^{2} 0^{m-5} 1^{m-5} 4 \\
(-1) 0^{m-3} 1^{m-6} 5\end{array}$ & $\begin{array}{l}(-1) 0^{m-3} 1^{m-9} 2^{4} \\
(-1) 0^{m-3} 1^{m-8} 2^{2} 3 \\
0^{m-1} 1^{m-9} 23^{2} \\
(-1) 0^{m-3} 1^{m-7} 3^{2} \\
0^{m-1} 1^{m-8} 25 \\
(-2) 0^{m-4} 1^{m-5} 4\end{array}$ & $\begin{array}{l}(-1)^{2} 0^{m-5} 1^{m-7} 2^{3} \\
(-2) 0^{m-4} 1^{m-7} 2^{3} \\
0^{m-1} 1^{m-9} 2^{2} 4 \\
(-2) 0^{m-4} 1^{m-6} 23 \\
0^{m-1} 1^{m-8} 34 \\
0^{m-1} 1^{m-7} 6\end{array}$ \\
\hline$m \geq 24$ & $2 m-8$ & $\begin{array}{l}0^{m-1} 1^{m-13} 2^{6} \\
(-1)^{3} 0^{m-7} 1^{m-7} 2^{3} \\
(-2) 0^{m-4} 1^{m-9} 2^{4} \\
0^{m-1} 1^{m-11} 2^{3} 4 \\
(-1) 0^{m-3} 1^{m-9} 23^{2} \\
(-1)^{2} 0^{m-5} 1^{m-7} 3^{2} \\
0^{m-1} 1^{m-10} 2^{2} 5 \\
(-1) 0^{m-3} 1^{m-8} 25 \\
(-2) 0^{m-4} 1^{m-7} 3^{2} \\
0^{m-1} 1^{m-9} 26 \\
(-1) 0^{m-3} 1^{m-7} 6 \\
0^{m-1} 1^{m-8} 7\end{array}$ & $\begin{array}{l}(-1) 0^{m-3} 1^{m-11} 2^{5} \\
0^{m-1} 1^{m-12} 2^{4} 3 \\
(-1)^{2} 0^{m-5} 1^{m-8} 2^{2} 3 \\
0^{m-1} 1^{m-11} 2^{2} 3^{2} \\
(-2) 0^{m-4} 1^{m-8} 2^{2} 3 \\
(-3) 0^{m-5} 1^{m-7} 2^{3} \\
0^{m-1} 1^{m-10} 234 \\
(-1) 0^{m-3} 1^{m-8} 34 \\
(-1)^{2} 0^{m-5} 1^{m-6} 5 \\
0^{m-1} 1^{m-9} 35 \\
(-2) 0^{m-4} 1^{m-6} 5\end{array}$ & $\begin{array}{l}(-1)^{2} 0^{m-5} 1^{m-9} 2^{4} \\
(-1) 0^{m-3} 1^{m-10} 2^{3} 3 \\
(-2)(-1) 0^{m-6} 1^{m-7} 2^{3} \\
(-1) 0^{m-3} 1^{m-9} 2^{2} 4 \\
(-1)^{2} 0^{m-5} 1^{m-7} 24 \\
(-2)(-1) 0^{m-6} 1^{m-6} 23 \\
0^{m-1} 1^{m-10} 3^{3} \\
(-2) 0^{m-4} 1^{m-7} 24 \\
(-3) 0^{m-5} 1^{m-6} 23 \\
0^{m-1} 1^{m-9} 4^{2} \\
(-3) 0^{m-5} 16^{m-5} 4\end{array}$ \\
\hline
\end{tabular}

\section{References}

[1] N. Alon and M. Dubiner, Zero-sum sets of prescribed size, in: Combinatorics, Paul Erdős is Eighty, Vol. 1, Bolyai Soc. Math. Stud., János Bolyai Math. Soc., Budapest, 1993, 33-50.

[2] N. Alon, M. B. Nathanson and I. Ruzsa, The polynomial method and restricted sums of congruence classes, J. Number Theory 56 (1996), 404-417. 
[3] A. Bialostocki and P. Dierker, On the Erdös-Ginzburg-Ziv theorem and the Ramsey numbers for stars and matchings, Discrete Math. 110 (1992), 1-8.

[4] A. Bialostocki and M. Lotspeich, Some developments of the Erdös-Ginzburg-Ziv Theorem. I, in: Sets, Graphs and Numbers (Budapest, 1991), Colloq. Math. Soc. János Bolyai 60, North-Holland, Amsterdam, 1992, 97-117.

[5] W. Brakemeier, Eine Anzahlformel von Zahlen modulo n, Monatsh. Math. 85 (1978), 277-282.

[6] H. Cao and Z. Sun, On sums of distinct representatives, Acta Arith. 87 (1998), 159-169.

[7] Y. Caro, Remarks on a zero-sum theorem, J. Combin. Theory Ser. A 76 (1996), $315-322$.

[8] H. Davenport, On the addition of residue classes, J. London Math. Soc. 10 (1935), $30-32$.

[9] J. A. Dias da Silva and Y. O. Hamidoune, A note on the minimal polynomial of the Kronecker sum of two linear operators, Linear Algebra Appl. 141 (1990), 283-287.

[10] R. B. Eggleton and P. Erdős, Two combinatorial problems in group theory, Acta Arith. 21 (1972), 111-116.

[11] P. Erdős, A. Ginzburg and A. Ziv, Theorem in additive number theory, Bull. Res. Council Israel 10F (1961), 41-43.

[12] P. Erdős and R. L. Graham, Old and new problems and results in combinatorial number theory, Monographie 28 de L'Enseignement Mathématique, Univ. de Genève, 1980.

[13] C. Flores and O. Ordaz, On the Erdös-Ginzburg-Ziv theorem, Discrete Math. 152 (1996), 321-324.

[14] L. Gallardo and G. Grekos, On Brakemeier's variant of the Erdös-Ginzburg-Ziv problem, Number Theory (Liptovský Ján, 1999), Tatra Mt. Math. Publ. 20 (2000), 91-98.

[15] L. Gallardo, G. Grekos, L. Habsieger, F. Hennecart, B. Landreau and A. Plagne, Restricted addition in $\mathbb{Z} / n$ and an application to the Erdös-Ginzburg-Ziv problem, J. London Math. Soc. (2) 65 (2002), 513-523.

[16] L. Gallardo, G. Grekos and J. Pihko, On a variant of the Erdös-Ginzburg-Ziv problem, Acta Arith. 89 (1999), 331-336.

[17] W. D. Gao, An addition theorem for finite cyclic groups, Discrete Math. 163 (1997), $257-265$.

[18] —, Addition theorems for finite abelian groups, J. Number Theory 53 (1995), 241246.

[19] W. D. Gao and Y. O. Hamidoune, Zero sums in abelian groups, Combin. Probab. Comput. 7 (1998), 261-263.

[20] D. Grynkiewicz, On a partition analog of the Cauchy-Davenport Theorem, preprint.

[21] D. Grynkiewicz and R. Sabar, Monochromatic and zero-sum sets of nondecreasing modified-diameter, preprint.

[22] Y. O. Hamidoune, A. S. Lladó and O. Serra, On restricted sums, Combin. Probab. Comput. 9 (2000), 513-518.

[23] Y. O. Hamidoune, O. Ordaz and A. Ortuño, On a combinatorial theorem of Erdös, Ginzburg and Ziv, Combin. Probab. Comput. 7 (1998), 403-412.

[24] J. H. B. Kemperman, On small sumsets in an abelian group, Acta Math. 103 (1960), 63-88.

[25] V. F. Lev, Restricted set addition in groups. I. The classical setting, J. London Math. Soc. (2) 62 (2000), 27-40. 
[26] M. B. Nathanson, Additive Number Theory. Inverse Problems and the Geometry of Sumsets, Grad. Texts in Math. 165, Springer, New York, 1996.

[27] R. Sabar, On a family of inequalities and the Erdös-Ginzburg-Ziv Theorem, preprint.

Department of Mathematics

Mathematics 253-37

University of Idaho

Caltech

Moscow, ID 83844, U.S.A.

Pasadena, CA 91125, U.S.A.

E-mail:math@uidaho.edu

Department of Mathematics

Albertson College

Caldwell, ID 83605, U.S.A.

E-mail: mlotspeich@albertson.edu

Received on 3.9.2002

and in revised form on 14.2.2003 